\title{
ON THE DIMENSION OF THE ZERO OR INFINITY TENDING SETS FOR LINEAR DIFFERENTIAL EQUATIONS
}

\author{
JAMES S. MULDOWNEY ${ }^{1}$
}

\begin{abstract}
ABSTRACr. There are well-known conditions which guarantee that all solutions to a system of $n$ differential equations $x^{\prime}=A(t) x, t \in[0, \omega)$, satisfy $\lim _{t \rightarrow \omega}|x(t)|=0$ $[\infty]$. Under certain stability assumptions on the system, Hartman [2], Coppel [1] and Macki and Muldowney [4] give necessary and sufficient [sufficient] conditions that the system has at least one nontrivial solution satisfying $\lim _{t \rightarrow \omega}|x(t)|=0[\infty]$. These results are extended by studying a sequence of matrices $A^{[k]}(t), k=$ $1, \ldots, n$, related to $A(t)$ such that, under the same stability assumptions as before, the given system has an $(n-k+1)$-dimensional zero [infinity] tending solution set if and only if [if] all nontrivial solutions of the system $y^{\prime}=A^{[k]}(t) y$ tend to zero [infinity].
\end{abstract}

Introduction. Consider the system of differential equations

$$
x^{\prime}=A(t) x, \quad t \in[0, \omega)
$$

where $A$ is an $n \times n$ matrix of complex-valued continuous functions on $[0, \omega)$ and $x$ is a column vector. In generalizing a result of Milloux [6] for second order scalar equations, it was shown by Hartman [2] (cf. [3, p. 501]) that if $0 \leqslant \lim _{t \rightarrow \omega}|x(t)|<$ $\infty$ exists for all solutions $x$, and $|\cdot|$ is the Euclidean norm, then there exists a nontrivial solution such that $\lim _{t \rightarrow \omega}|x(t)|=0$ if and only if $\lim _{t \rightarrow \omega} \int_{0}^{t} \operatorname{Re} \operatorname{Tr} A=$ $-\infty$. Coppel $[1$, p. 60] showed that the result holds for any norm. Macki and Muldowney [4] weakened the restriction that $|x(t)|$ tends to a limit to the stability requirement I below. Analogous results on the existence of infinity tending solutions were also obtained in $[1,2,4]$.

Results. It will be assumed that either I or II holds for (1).

I. (i) All solutions satisfy lim $\sup _{t \rightarrow \omega}|x(t)|<\infty$.

(ii) If $\lim \inf _{t \rightarrow \omega}|x(t)|=0$, then $\lim _{t \rightarrow \omega}|x(t)|=0$.

II. If $\lim \sup _{t \rightarrow \omega}|x(t)|=\infty$, then $\lim _{t \rightarrow \omega}|x(t)|=\infty$.

The condition $I$ is more restrictive than Lyapunov stability of (1) and is implied by uniform stability; cf. [4]. I holds in particular if $\lim _{t \rightarrow \omega}|x(t)|<\infty$ exists for all solutions and II holds if $\lim _{t \rightarrow \omega}|x(t)| \leqslant \infty$ exists for all solutions. Concrete conditions which imply I or II are given later in the paper.

If $X=\left[x_{i}^{j}\right]$ is any $n \times m$ matrix and $1<k<\min \{n, m\}$, the $k$ th compound $X^{(k)}$ of $X$ is the $\left(\begin{array}{c}n \\ k\end{array}\right) \times\left(\begin{array}{c}m \\ k\end{array}\right)$ matrix whose entries are $x_{i_{1}}^{j_{1}} \ldots j_{i_{k}}$, the minors of $X$

Received by the editors June 25, 1980.

1980 Mathematics Subject Classification. Primary 34A30; Secondary 34D05.

1 Research supported by NSERC Grant A-7197. 
determined by the rows $\left(i_{1} \ldots i_{k}\right)$ and the columns $\left(j_{1} \ldots j_{k}\right), 1<i_{1}<i_{2}$ $<\cdots<i_{k} \leqslant n, 1 \leqslant j_{1}<j_{2}<\cdots<j_{k} \leqslant m$. These $k$-tuples taken in lexicographic order index the rows and columns respectively of $X^{(k)}$. Thus, for example if $m=n, X^{(1)}=\left[x_{i}^{j}\right]=X$ and $X^{(n)}=x_{12}^{12} \ldots n=\operatorname{det} X$. A discussion of compound matrices may be found in [5 and 8].

If $A=\left[a_{i}^{j}\right]$ is an $n \times n$ matrix and $1 \leqslant k<n$, then $A^{[k]}$ is the $\left(\begin{array}{c}n \\ k\end{array}\right) \times\left(\begin{array}{c}n \\ k\end{array}\right)$ matrix defined as follows.

(a) For any integer $p=1, \ldots,\left(\begin{array}{c}n \\ k\end{array}\right)$ and ordered $k$-tuple of integers $\left(p_{1} \ldots p_{k}\right)$, $1 \leqslant p_{1}<p_{2}<\cdots<p_{k} \leqslant n$, write $(p)=\left(p_{1} \ldots p_{k}\right)$ if $\left(p_{1} \ldots p_{k}\right)$ is the $p$ th $k$ tuple in the lexicographic order.

(b) Let $(p)=\left(p_{1} \ldots p_{k}\right)$ and $(q)=\left(q_{1} \ldots q_{k}\right)$. If there are two or more entries in $(p)$ which do not occur in $(q)$, then the entry in the $p$ th row and $q$ th column of $A^{[k]}$ is 0 . If there is exactly one entry $p_{s}$ in $(p)$ which does not occur in $(q)$ and $q_{r}$ in (q) does not occur in $(p)$, then the entry in $p$ th row and $q$ th column of $A^{[k]}$ is $(-1)^{r+s} a_{p_{s}}^{q_{r}}$. If $(p)=(q)$, then the corresponding diagonal element is $a_{p_{1}}^{p_{1}}+a_{p_{2}}^{p_{2}}$ $+\cdots+a_{p_{k}}^{p_{k}}$. For example, if $A$ is any $n \times n$ matrix, then $A^{[1]}=A, A^{[n]}=a_{1}^{1}$ $+\cdots+a_{n}^{n}=\operatorname{Tr} A$.

We will also consider the equation

$$
y^{\prime}=A^{[k]}(t) y, \quad k=1, \ldots, n, \quad t \in[0, \omega) .
$$

The relationship between (1) and (k) is established by Lemma 1 . The equations $(\mathrm{k})$ were first studied by Schwarz [7], where further examples of the matrices $A^{[k]}$ may be found.

LEMMA 1. If $X(t)$ is an $n \times m$ [fundamental] matrix solution of $(1)$, then $X^{(k)}(t)$ is an $\left(\begin{array}{l}n \\ k\end{array}\right) \times\left(\begin{array}{c}m \\ k\end{array}\right)$ [ fundamental] matrix solution of $(\mathbf{k})$.

PROof. From the rule for differentiating determinants and (1)

$$
\begin{aligned}
\frac{d}{d t} x_{i_{1} \ldots i_{k}}^{j_{1} \ldots j_{k}} & =\sum_{s=1}^{k} \sum_{r=1}^{n} a_{i_{s}}^{r} x_{i_{1} \ldots r}^{j_{1} \ldots j_{s} \ldots j_{k}} \ldots i_{k} \\
& =\left(\sum_{s=1}^{k} a_{i_{s}}^{i_{s}}\right) x_{i_{1} \ldots i_{k}}^{j_{1} \ldots j_{k}}+\sum_{s=1}^{k} \sum_{r \notin(i)} a_{i_{s}}^{r} x_{i_{1} \ldots j_{s} \ldots j_{k}}^{j_{1} \ldots j_{k}}
\end{aligned}
$$

and therefore $d X^{(k)} / d t=A^{[k]} X^{(k)}$, as asserted. Also, if $X$ is fundamental, then $m=n$ and $X^{(k)}$ is fundamental since a theorem of Sylvester states $\operatorname{det}\left[X^{(k)}\right]=$ [det $X]^{\alpha}$, where $\alpha=\left(\begin{array}{c}n-1 \\ k-1\end{array}\right)$ (cf. [5, p. 17 or 8, p. 64]).

LemMa 2. If (1) satisfies Condition I then (k) satisfies Condition I.

Proof. Suppose Condition I holds for (1). Clearly Condition I(i) also holds for (k). To prove that I(ii) holds for (k), first observe that the solution space of (1) is the direct sum of the set of zero-tending solutions of (1) and a supplementary subspace of solutions which are bounded but do not tend to zero. Thus a fundamental matrix $X(t)$ for (1) exists in which the first $n-m$ columns span the zero-tending set and the last $m$ columns span the supplementary subspace, $0<m$ $<n$. It follows from Lemma 1 that if $m=0$ then every solution $y(t)=X^{(k)}(t) \xi$ 
satisfies $\lim _{t \rightarrow \omega} y(t)=0$ and (k) satisfies Condition I(ii). If $m>0$, let $V(t), Z(t)$ be the $n \times(n-m), n \times m$ matrices formed respectively from the first $n-m$ and last $m$ columns of $X(t)$. Thus any solution $y$ of $(\mathrm{k})$ may be expressed in the form

$$
y(t)=X^{(k)}(t) \xi=W(t) \eta+Z^{(k)}(t) \zeta
$$

where $W(t)$ is an $\left(\begin{array}{c}n \\ k\end{array}\right) \times\left[\left(\begin{array}{c}n \\ k\end{array}\right)-\left(\begin{array}{c}m \\ k\end{array}\right)\right]$ matrix in which each entry is a $k \times k$ minor of $X(t)$ of which at least one column consists of entries from a column of $V(t)$. Thus $\lim _{t \rightarrow \omega} W(t) \eta=0$ for all $\eta$. It will be shown that, if $\lim _{i \rightarrow \omega}|y(t)|=0$, then $\zeta=0$ and therefore $\lim _{t \rightarrow \omega} y(t)=0$ so that (k) satisfies I(ii). To see this, observe that if $\lim \inf _{t \rightarrow \omega}|y(t)|=0$, then $\lim _{\inf _{t \rightarrow \omega}}|z(t)|=0$ where $z(t)=Z^{(k)}(t) \zeta$. Thus there exists a sequence $t_{i} \in[0, \omega)$ such that $\lim _{i \rightarrow \infty} t_{i}=\omega, \lim _{i \rightarrow \infty} z\left(t_{i}\right)=0$ and $\lim _{i \rightarrow \infty} Z\left(t_{i}\right)=C$ exists. Then

$$
C^{(k)} \zeta=\lim _{i \rightarrow \infty} Z^{(k)}\left(t_{i}\right) \zeta=\lim _{i \rightarrow \infty} z\left(t_{i}\right)=0 .
$$

If $\zeta \neq 0$, then the $\left(\begin{array}{c}n \\ k\end{array}\right) \times\left(\begin{array}{c}m \\ k\end{array}\right)$ matrix $C^{(k)}$ has less than maximal rank which, by Sylvester's Theorem implies that $C$ has less than maximal rank. Thus there is a nonzero vector $\rho$ such that

$$
0=C \rho=\lim _{i \rightarrow \infty} Z\left(t_{i}\right) \rho=\lim _{t \rightarrow \infty} Z(t) \rho
$$

by Condition I(ii) for (1), which contradicts the definition of $Z$. Thus $\zeta=0$ as asserted.

The author is indebted to Professor Binyamin Schwarz for pointing out an error in the original proof of Lemma 2.

THEOREM. (a) Suppose the system (1) satisfies Condition I. Then (1) has an $(n-k+1)$-dimensional set of solutions $x$ satisfying $\lim _{t \rightarrow \omega}|x(t)|=0$ if all solutions $y$ of (k) satisfy $\lim \inf _{t \rightarrow \omega}|y(t)|=0$ and only if all $y$ satisfy $\lim _{t \rightarrow \omega}|y(t)|=0$.

(b) Suppose (1) satisfies II. Then (1) has an $(n-k+1)$-dimensional set of solutions $x$ satisfying $\lim _{t \rightarrow \omega}|x(t)|=\infty$ if all nontrivial solutions $y$ of $(\mathbf{k})$ satisfy $\lim \sup _{t \rightarrow \omega}|y(t)|=\infty$.

In the case $k=n$ this theorem is Theorem 1 of [4].

Proof of (a). Let $X(t)$ be a fundamental matrix for (1). Condition I(i) implies there exists a sequence of points $t_{i} \in[0, \omega)$ such that $\lim _{i \rightarrow \infty} t_{i}=\omega$ and $\lim _{i \rightarrow \infty} X\left(t_{i}\right)=C$ exists. Thus each solution $x(t)=X(t) \xi$ of (1) satisfies $\lim _{i \rightarrow \infty} x\left(t_{i}\right)=C \xi$. From this it follows that if all solutions of (k) satisfy $\lim \inf _{t \rightarrow \omega}|y(t)|=0$, then the lemmas imply

$$
0=\lim _{t \rightarrow \omega} X^{(k)}(t)=\lim _{i \rightarrow \infty} X^{(k)}\left(t_{i}\right)=C^{(k)}
$$

i.e., the rank of $C$ is at most $k-1$. Therefore there is a solution set of dimension at least $n-k+1$ such that $\lim _{i \rightarrow \infty} x\left(t_{i}\right)=0$ and hence $\lim _{i \rightarrow \omega}|x(t)|=0$. Now Condition I(ii) shows that (1) has a solution set of dimension at least $n-k+1$ satisfying $\lim _{t \rightarrow \omega}|x(t)|=0$.

To prove the necessity of the condition in Part (a), suppose that (1) has an $(n-k+1)$-dimensional set of zero tending solutions. Then any set of $k$ linearly 
independent solutions contains a zero tending solution in its span and hence, from I(i), $\lim _{t \rightarrow \omega} X^{(k)}(t)=0$ so that all solutions $y$ of $(\mathrm{k})$ satisfy $\lim _{t \rightarrow \omega}|y(t)|=0$.

Proof OF (b). Suppose all nontrivial solutions $y$ of (k) satisfy $\lim \sup _{t \rightarrow \omega}|y(t)|=$ $\infty$. Thus, if $X(t)$ is a fundamental matrix of (1), every column of $X^{(k)}(t)$ contains an unbounded entry which implies that every set of $k$ independent solutions to (1) contains an unbounded solution. Thus the subspace of bounded solutions has dimension at most $k-1$ and a supplementary subspace, except for the solution $x=0$, consists entirely of unbounded solutions and has dimension at least $n-k$ +1 . From II of these solutions satisfy $\lim _{t \rightarrow \omega}|x(t)|=\infty$.

It remains to provide some concrete criteria for the conditons of the theorem to hold. The Lozinskin measure (cf. [1, pp. 41, 58]) of a matrix $A$ is defined by

$$
\mu(A)=\lim _{h \rightarrow 0^{+}} \frac{1}{h}[|I+h A|-1]
$$

and has the property that, for each solution $x$ of (1),

$$
|x(t)| \exp \left(-\int_{t_{0}}^{t} \mu[A]\right), \quad|x(t)| \exp \left(\int_{t_{0}}^{t} \mu[-A]\right)
$$

are nonincreasing and nondecreasing functions of $t$ respectively. The following observations on (1) may be deduced.

III. If $\lim _{t \rightarrow \omega}\left[\lim \inf _{t \rightarrow \omega}\right] \int_{0}^{t} \mu(A)=-\infty$, then $\lim _{t \rightarrow \omega}\left[\lim \inf _{t \rightarrow \omega}\right]|x(t)|=0$ for all solutions.

IV. If $\lim _{t \rightarrow \infty}\left[\lim \inf _{t \rightarrow \omega}\right] \int_{0}^{t} \mu(-A)=-\infty$, then $\lim _{t \rightarrow \omega}\left[\lim \sup _{t \rightarrow \omega}\right]|x(t)|=\infty$ for all nontrivial solutions.

V. If $\int_{t_{0}}^{t} \mu(A) \leqslant M$ for all $t_{0}, t, 0 \leqslant t_{0} \leqslant t<\omega$, then (1) satisfies Condition I.

VI. If $\int_{t_{0}}^{t} \mu(-A) \leqslant M$ for all $t_{0}, t, 0 \leqslant t_{0} \leqslant t<\omega$, then (1) satisfies Condition II.

The value of $\mu(A)$ depends on the norm $|\cdot|$ used. In the cases $|x|=\sup _{i}\left|x_{i}\right|$, $\Sigma_{i}\left|x_{i}\right|,\left[\Sigma\left|x_{i}\right|^{2}\right]^{1 / 2}$, the Lozinskin measure is given by

$$
\mu(A)=\sup _{i}\left(\operatorname{Re} a_{i}^{i}+\sum_{j \neq i}\left|a_{i}^{j}\right|\right), \quad \sup _{i}\left(\operatorname{Re} a_{i}^{i}+\sum_{j \neq i}\left|a_{j}^{i}\right|\right), \quad \lambda_{1}
$$

respectively, where $\lambda_{1} \geqslant \lambda_{2} \geqslant \cdots \geqslant \lambda_{n}$ are the eigenvalues of $\frac{1}{2}\left(A^{*}+A\right)$. Here $A^{*}$ is the Hermitian transpose of $A$. It is also easy to write down $\mu\left(A^{[k]}\right)$ in these cases which are given by

$$
\mu\left(A^{[k]}\right)=\left\{\begin{array}{l}
\sup _{(i)}\left[\operatorname{Re}\left(a_{i_{1}}^{i_{1}}+\cdots+a_{i_{k}}^{i_{k}}\right)+\sum_{j \notin(i)}\left(\left|a_{i_{1}}^{j}\right|+\cdots+\left|a_{i_{k}}^{j}\right|\right)\right], \\
\sup _{(i)}\left[\operatorname{Re}\left(a_{i_{1}}^{i_{1}}+\cdots+a_{i_{k}}^{i_{k}}\right)+\sum_{j \notin(i)}\left(\left|a_{j}^{i_{1}}\right|+\cdots+\left|a_{j}^{i_{k}}\right|\right)\right], \\
\lambda_{1}+\cdots+\lambda_{k},
\end{array}\right.
$$

respectively. Also, it is clear that $\mu\left(A^{[n]}\right)=\operatorname{Re} \operatorname{Tr} A$ since $A^{[n]}=\operatorname{Tr} A$.

Corollary. Suppose that

(i)

$$
\int_{t_{0}}^{t} \mu(A) \leqslant M \quad\left[\int_{t_{0}}^{t} \mu(-A) \leqslant M\right] \text { for all } t_{0}, t, 0<t_{0}<t<\omega
$$


(ii)

$$
\liminf _{t \rightarrow \omega} \int_{0}^{t} \mu\left(A^{[k]}\right)=-\infty\left[\liminf _{t \rightarrow \omega} \int_{0}^{t} \mu\left(-A^{[k]}\right)=-\infty\right] .
$$

Then (1) has a set of solutions $x$ of dimension at least $(n-k+1)$ for which $\lim _{t \rightarrow \omega}|x(t)|=0\left[\lim _{t \rightarrow \omega}|x(t)|=\infty\right]$.

This corollary follows from III, V [IV, VI] and the theorem.

EXAMPLE. Consider

$$
A(t)=\left[\begin{array}{ccc}
-1 & \frac{1}{2} & \frac{1}{2} \\
\frac{t}{2} & -t & \frac{t}{2} \\
\frac{t^{2}}{2} & \frac{t^{2}}{2} & -t^{2}
\end{array}\right], \quad t \geqslant 1
$$

Then, for the norm $|x|=\sup _{i}\left|x_{i}\right|$,

$$
\mu(A)=0, \quad \mu\left(A^{[2]}\right)=-\frac{1}{2}(1+t), \quad \mu\left(A^{[3]}\right)=-\left(1+t+t^{2}\right) .
$$

From the corollary, (1) has a set of solutions $x$ of dimension at least 2 for which $\lim _{t \rightarrow \infty}|x(t)|=0$. In fact, the dimension is exactly 2 since $x(t)=(1,1,1)^{*}$ is a constant solution.

It may be observed that interesting asymptotic information about (1) may be obtained by combining changes of variable with the results in this paper as was done in detail in [4] in the case $k=n$.

\section{REFERENCES}

1. W. A. Coppel, Stability and asymptotic behavior of differential equations, Heath, Boston, Mass., 1965.

2. P. Hartman, The existence of large or small solutions of linear differential equations, Duke Math. J. 28 (1961), 421-429.

3.

4. J. W. Macki and J. S. Muldowney, The asymptotic behaviour of solutions to linear systems of ordinary differential equations, Pacific J. Math. 33 (1970), 693-706.

5. M. Marcus and H. Minc, A survey of matrix theory and matrix inequalities, Allyn and Bacon, Boston, Mass., 1964.

6. H. Milloux, Sur l'équation différentielle $x^{\prime \prime}+A(t) x=0$, Prace Mat. Fiz. 41 (1934), 39-53.

7. B. Schwarz, Totally positive differential systems, Pacific J. Math. 32 (1970), 203-229.

8. M. Ō. Tnūthail, Algēbar Iolscoile, Oifig an tSolāthair, Baile Âtha Cliath, 1947.

Department of Mathematics, University of Alberta, Edmonton, Alberta T6g 2G1, Canada 